\title{
Evaluation of microRNA 92a Expression and Its Target Protein Bim in Colorectal Cancer
}

\author{
Ahmed Zaki ${ }^{1 *}$, Amal Fawzy ${ }^{1}$, Samia Y Akel ${ }^{1}$, H Gamal ${ }^{2}$, Reham A A Elshimy ${ }^{1}$
}

\begin{abstract}
Background: Colorectal cancer is one of the most commonly diagnosed cancers and leading causes of malignancy-related deaths all over the world. MicroRNAs (miRNAs) can regulate more than 60\% of human genes, including tumor-stimulating, and -suppressor genes. Therefore, they can promote cancer development and affect risk of malignancy. miR-92a overexpression in CRC enhances tumor proliferation, invasion, and metastasis through downregulating different pro-apoptosis proteins including Bim. This study aimed to assess the role of plasma miR-92a as non-invasive marker in CRC patients, outline correlation between plasma miR-92a and serum Bim, and determine their correlations with clinicopathological parameters in CRC and adenoma patients. Methods: A total of 54 newly diagnosed CRC patients, 15 colonic adenoma patients, and 15 age- and sex-matched control subjects were recruited in this study. Plasma miR-92a was assayed by TaqMan qRT-PCR and serum Bim was measured by ELISA. Results: Statistically significant overexpression of serum miR-92a was observed in CRC patients as compared to adenoma and control groups $(\mathrm{p}<0.001 \mathrm{each})$ and lower serum Bim in CRC patients as compared to adenoma and control groups ( $\mathrm{p}=0.001, \mathrm{p}$ $<0.001$ respectively). The ROC curve analysis showed excellent AUC for plasma miR-92a in discriminating CRC from control ( $\mathrm{AUC}=0.994)$, and adenoma $(\mathrm{AUC}=0.993)$ groups with highest diagnostic performance in discriminating $\mathrm{CRC}$ from controls (at cutoff 1.43 , sensitivity $98.1 \%$, specificity $93.9 \%$ ), and adenoma patients (at cutoff 1.78 , sensitivity $92.6 \%$, specificity 93.3\%). The diagnostic performance in discriminating early from late CRC was good (at cutoff 15 , $\mathrm{AUC}=0.641$, sensitivity $61.2 \%$, specificity $80 \%$ ). A significant negative correlation was evident between plasma miR-92a and serum Bim both in adenoma and CRC groups $(\mathrm{P}<0.001$ for both). Higher plasma miR-92a expression $(\mathrm{r}=0.275$, $\mathrm{p}=0.044)$ and lower serum $\operatorname{Bim}(\mathrm{r}=-0.299, \mathrm{p}=0.028)$ were found to be correlated with late CRC stages. Conclusion: Circulating miR-92a and Bim could be promising, non-invasive diagnostic and prognostic biomarkers in $\mathrm{CRC}$.
\end{abstract}

Keywords: miR-92a- Bim- CRC

Asian Pac J Cancer Prev, 23 (2), 723-730

\section{Introduction}

Colorectal cancer (CRC) is the third most prevalent cancer in males and second in females worldwide. Regarding mortality statistics, it represents the third leading cause of malignancy-related deaths in women and men, and the second prevalent cause of cancer deaths when men and women are combined together. Almost $50 \%$ of CRC patients present with metastasis at diagnosis time or develop it during disease progression (Cekaite et al., 2016).

In Egypt, the incidence rate of CRC is 5.1\% in men and $4.7 \%$ in women. Egypt is having high global incidence of early CRC as $35 \%$ of Egyptian CRC patients were below age of 40 (Bader et al., 2020).

The currently used CRC diagnostic procedures and lab tests are either invasive and discomforting or show significantly low specificity and sensitivity (Ferrari et al., 2021), and that's why extensive research efforts are directed towards the finding of less invasive, more convenient, and cost-effective biomarkers with higher diagnostic accuracy to detect CRC patients at earlier resectable stages.

MicroRNAs (miRNAs) are small non-coding RNAs, 18-23 nucleotides long, which act as post-transcriptional regulators of gene expression (Qadir et al., 2019).

miR-92a gene is considered an integral part of miR-17-92 precursor cluster positioned at chromosome 13q31.3 (Mogilyansky and Rigoutsos, 2013). miR-92a and its currently determined gene targets have been involved in cell cycle control and cell signaling, and thus very necessary during all stages of mammalian development (Fang et al., 2017). Many studies have concluded that miR-92a is abnormally overexpressed in different malignancies including leukemia, colorectal, lung, and ovarian cancers (Mogilyansky and Rigoutsos, 2013). 
BCL2 interacting mediator of cell death (Bim) protein is one of the most important pro-apoptotic factors extensively expressed in normal tissues. The activated Bim could stimulate activation of Bax protein leading to apoptosis through mitochondrial pathway ( $\mathrm{Li}$ et al., 2014). It has been reported as a target for miR-92a and its downregulation relives its tumor-suppressing activity and thought to have a vital role in promoted CRC angiogenesis and proliferation (Xuan et al., 2015; Sionov et al., 2015).

Up to our knowledge, this is the first study that correlates serum Bim levels with plasma miR-92a expression in CRC and colonic adenoma patients. This study aims to assess plasma miR-92a expression in CRC patients and outline correlation between plasma miR-92a expression and serum Bim protein in CRC patients and their clinicopathological parameters.

\section{Materials and Methods}

\section{Patients}

This study was done on 84 participants, including 54 newly diagnosed CRC Egyptian patients at different stages, 15 colonic adenoma Egyptian patients, and 15 age- and sex-matched controls. Patients were recruited from surgical oncology outpatient clinics at National Cancer Institute (NCI) hospital, Cairo University, from September 2016 till March 2018. The study was permitted by the Institutional Review Board (IRB) of the NCI, Cairo University. It was permitted according to Helsinki guidelines of studies performed on human beings and written consent was obtained from all study subjects before their enrollment. All patients were subjected to; full history taking and clinical examination, ordinary biochemical and markers investigations, imaging techniques in the form of: CT and MRI, and endoscopic studies with biopsy taking and histopathological examination. Samples were obtained from all patients prior to any therapeutic or surgical intervention. Participants' age showed as mean \pm SD of $(50.7 \pm 15)$ years in CRC patients, (37.9 \pm 12.5$)$ years in the adenoma patients, and $(44.6 \pm 12.4)$ years in controls.

\section{Sample Collection}

Eight milliliters of venous blood were withdrawn under aseptic precautions, 4 millimeters were put into sterile vaccutainer tube containing K2 EDTA and centrifuged at $3400 \mathrm{rpm}$ for $15 \mathrm{~min}$ at $4 \mathrm{oc}$. Supernatant plasma was transferred to sterile, labeled Eppendorf tube. These plasma samples were stored at $-20^{\circ} \mathrm{C}$ until RNA extraction followed by assay of miR-92a by qRT-PCR. The other 4 milliliters were allowed to clot for 30 minutes at room temperature before centrifugation for 15 minutes at $3400 \mathrm{rpm}$. the supernatant serum was collected and stored at -20oc until ELISA assay of all samples for Bim concentration and determination of serum CEA, CA 19.9 using Cobas e411 Autoanalyzer, Roche.

Analysis of expression level of miR-92a by $q R T-P C R$ according to TaqMan protocol
Total RNA, including microRNAs, was extracted using the miRNeasy Mini Kit catalog\#: 217184 (Qiagen, Germany), according to manufacturer's instructions.

Five $\mu \mathrm{L}$ of extracted total RNA were used as template for synthesis of cDNA performed using TaqMan ${ }^{\circledR}$ MicroRNA Reverse Transcription Kit (cat. no. 4366596) and TaqMan ${ }^{\circledR}$ MicroRNA Assay Primers manufactured by Thermo Scientific, USA. $15 \mu \mathrm{L}$ of reverse transcription reaction components were used as total volume. Each $15 \mu 1$ reaction consists of $7 \mu 1$ master mix, $3 \mu 1$ of miR-92a primer $(5 x)$, and $5 \mu$ l of RNA sample.

The extracted RNA was quantified and its purity assessed using NanoDrop R (ND)-1000 spectrophotometer (NanoDrop Technology, WILM, USA). RNA concentration in samples was detected via absorbance at $260 \mathrm{~nm}$ with mean concentration $13 \mu \mathrm{g} / \mathrm{ml}(\mathrm{A} 260=2-21 \mu \mathrm{g} / \mathrm{ml})$, while purity was determined via absorbance ratios at 260/280 with accepted range at (1.8-2.1).

The miR-92a expression was measured by qRT-PCR using TaqMan ${ }^{\circledR}$ microRNA assay kit and $\operatorname{TaqMan}^{\circledR}$ Universal PCR Master Mix II kit catalog No. 4428175 (Applied Biosystems). We pipetted $10 \mu 1$ of TaqMan $^{\circledR}$ Universal PCR Master Mix II, $1 \mu$ l of genespecific TaqMan ${ }^{\circledR}$ primer/probe mix, and $6.5 \mu 1$ of nuclease-free water. Then, $2.5 \mu \mathrm{l}$ of each cDNA were pipetted in the corresponding well to reach final volume of $20 \mu \mathrm{l} /$ well.

TaqMan qRT-PCR was performed in duplicate, and U6 snRNA was used as endogenous (reference) gene for normalizing miR-92a expression. The relative miR-92a expression was calculated using equation $2^{-\Delta \Delta \mathrm{CT}}$ where $\Delta \Delta \mathrm{CT}=(\mathrm{CT}$ miR-92a - CT U6) patient sample - (CT miR-92a - CT U6) control sample (Livak and Schmittgen, 2001).

Primer sequences: for miR-92a, forward-5' ACACAGGTTGGGATCGGTTG-3', and reverse5'-CAAACTCAACAGGCCGGGA-3'; and for U6, forward-5'-CTCGCTTCGGCAGCACA-3', and reverse5'-AACGCTTCACGAATTTGCGT-3'.

\section{Quantitative determination of Bim in serum samples by ELISA technique}

Serum Bim was measured using sandwich ELISA Kit (catalog\#: E-EL-H0582) supplied by eBioscience Corp. (ThermoFisher Scientific, USA) according to manufacturer's instructions.

\section{Statistical Methods}

In this study, data was tested using SPSS version 25 (SPSS Inc., Chicago, IL, USA). Numerical parametric data was expressed as mean $\pm \mathrm{SD}$, while non-parametric variables as median and range. Qualitative variables were expressed as frequency and percentage. Student $\mathrm{T}$ test was used to assess the difference between 2 study groups means. The difference of non-parametric variable between 2 study groups was assessed using Mann-Whitney U Test. To examine relation between qualitative variables, Chi-square or Fisher's exact test was used. Kruskal-Wallis test was used for assessing difference between more than two independent variables. To find correlation between two variables, Spearman's rho (r) was calculated. The 
ROC curve was used to determine cut-off values and to analyze diagnostic utility of different markers. All p-values are tow-sided and values $<0.05$ was considered statistically significant.

\section{Results}

Comparison of studied markers 'levels between all studied groups and according to clinicopathological parameter

This study was carried out on 54 CRC patients aged $(50.7 \pm 15)$ years. Male patients were $28(51.9 \%)$, while female patients were $26(48.1 \%)$. Based on radiologic and colonoscopic and operative findings, the most common CRC site was the colon $(27 ; 50 \%)$, followed by rectum $(18 ; 33.3 \%)$, the mean tumor size was $(7.8 \pm 2.5 \mathrm{~cm})$, the late CRC stages (III+IV) were the predominant (49; $90.7 \%$ ), and the most prevalent histological grades were grade II $(44 ; 81.5 \%)$ followed by grade III $(8 ; 14.8 \%)$ as shown in Table 1.

Plasma miR-92a represented as (median; range), showed significant upregulation in CRC patients (31.1; 1.2-9753.8) when compared with control $(0.5 ; 0-2.3)$ and adenoma $(0.6 ; 0.01-1.2)$ groups $(\mathrm{p}<0.001$ for both). No significant difference was found in plasma miR-92a expression between adenoma and control groups $(\mathrm{p}=0.787)$ as shown in Table 1 and Figure 1. Despite the increase in miR-92a expression pattern from early (10.9; 2.4-402.9) to late (36.6; 1.2-9753.8) CRC stages, there was no significant difference found in its expression $(\mathrm{p}=0.303)$. Moreover, no significant difference in miR-92a expression found according to gender, Family History, tumor site, side of colonic tumor and pathological grade in $\mathrm{CRC}$ and adenoma groups as shown in Table 1.

Serum Bim represented as (median; range), showed
(A)

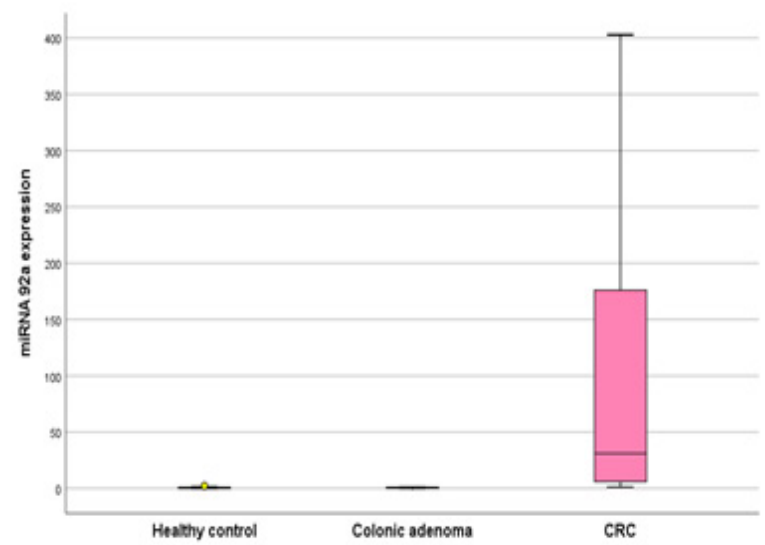

(B)

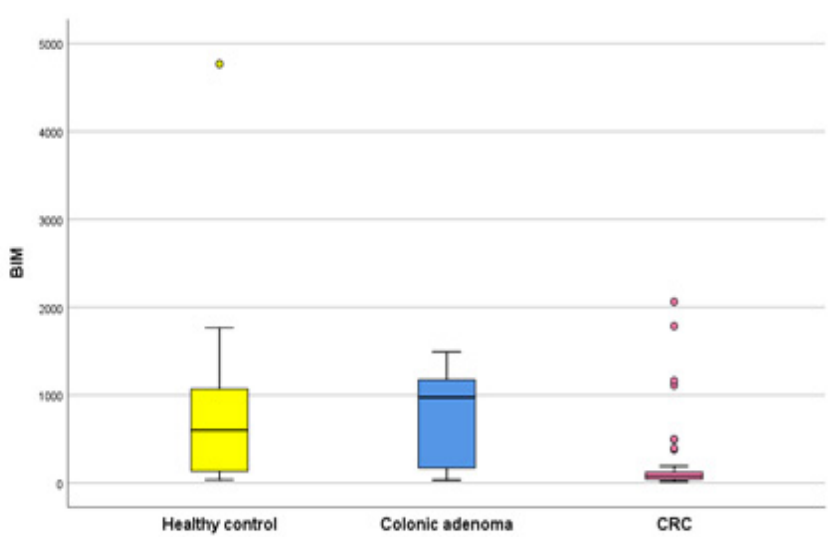

Figure 1. Box Plots for miR-92, Bim in CRC Patients Versus Adenoma Patients and Controls. miR-92a expression is significantly higher in CRC (A) compared to adenoma \& control groups $(p<0.001$ each). Significantly lower Bim (B) in CRC compared to adenoma patients $(\mathrm{p}=0.001)$ and controls $(\mathrm{p}<0.001)$.

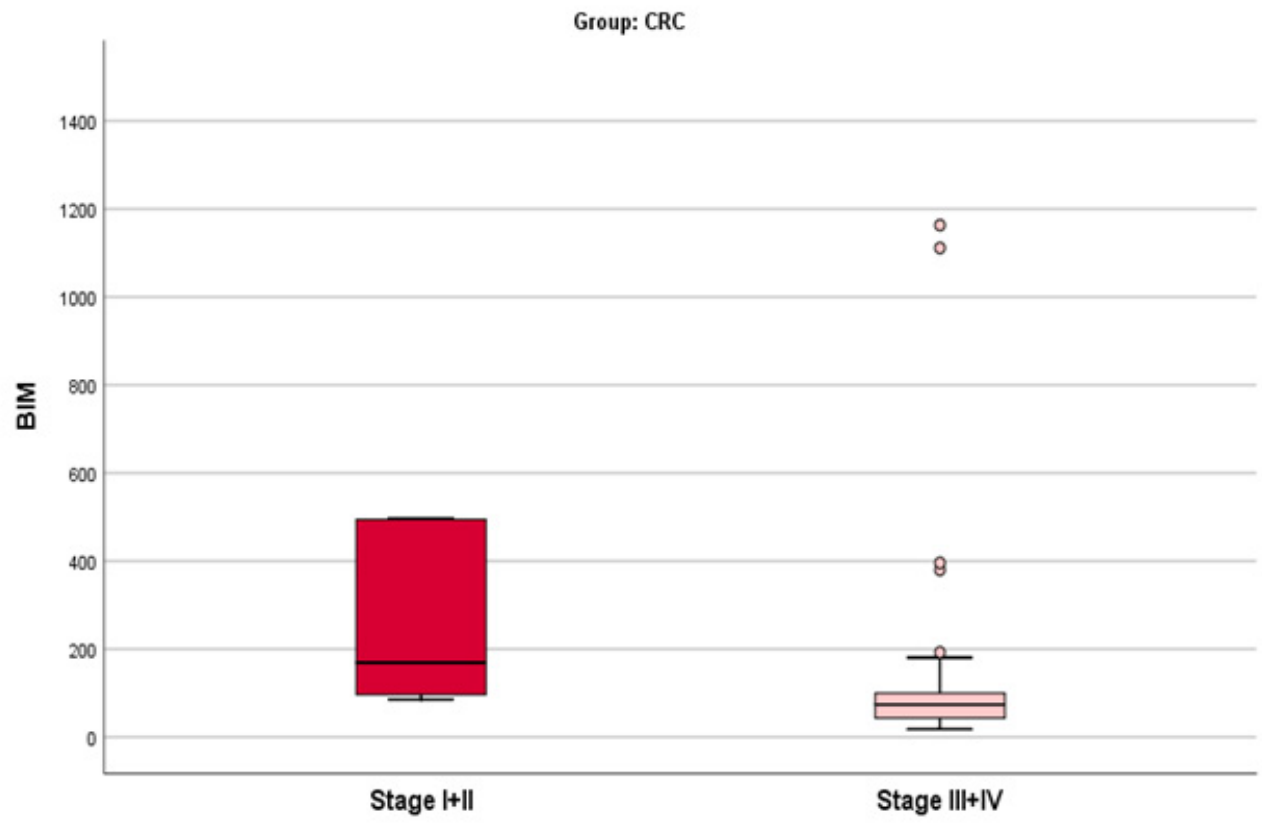

Figure 2. Serum Bim Concentration According to Stage in CRC Group. 


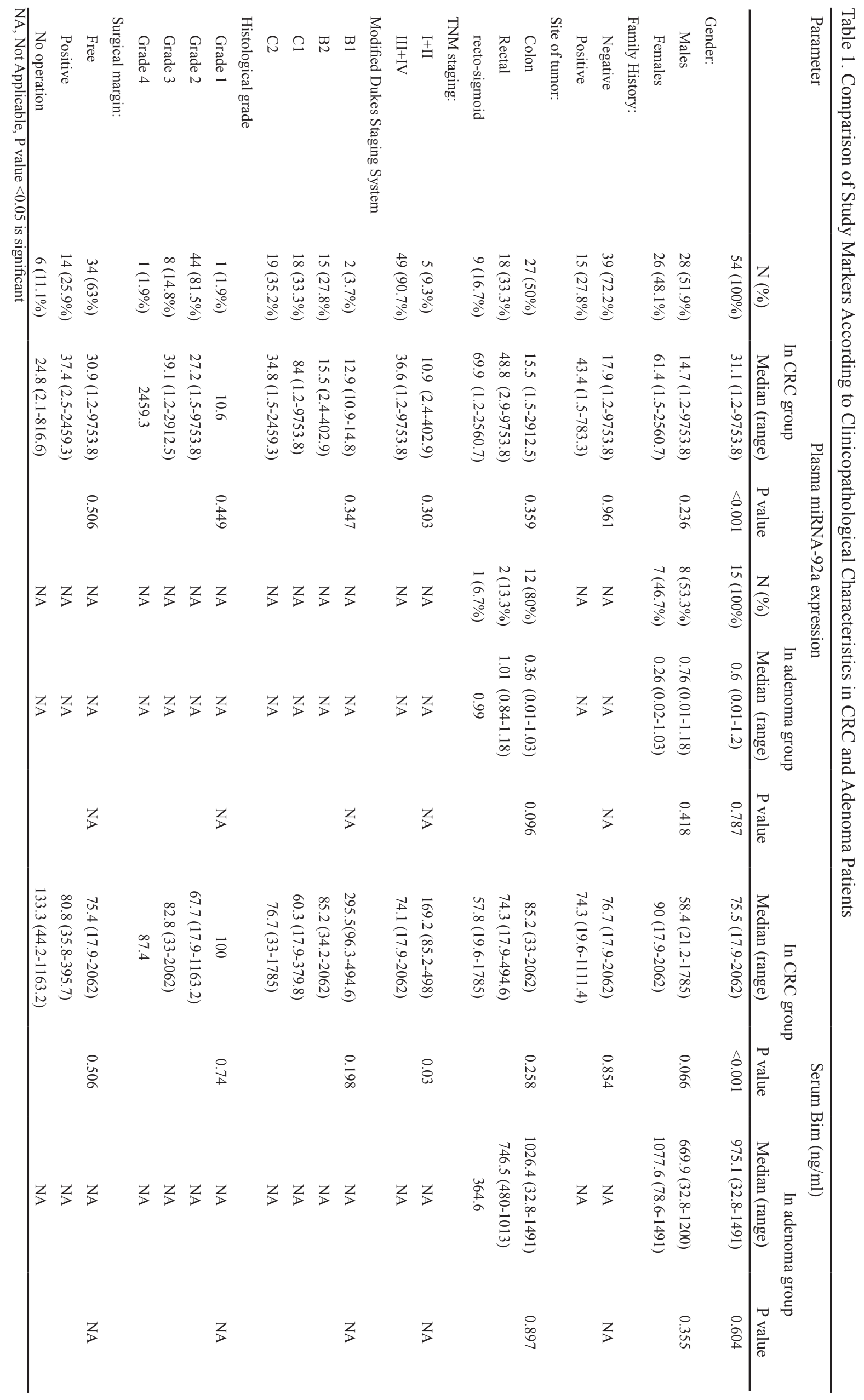


(A)

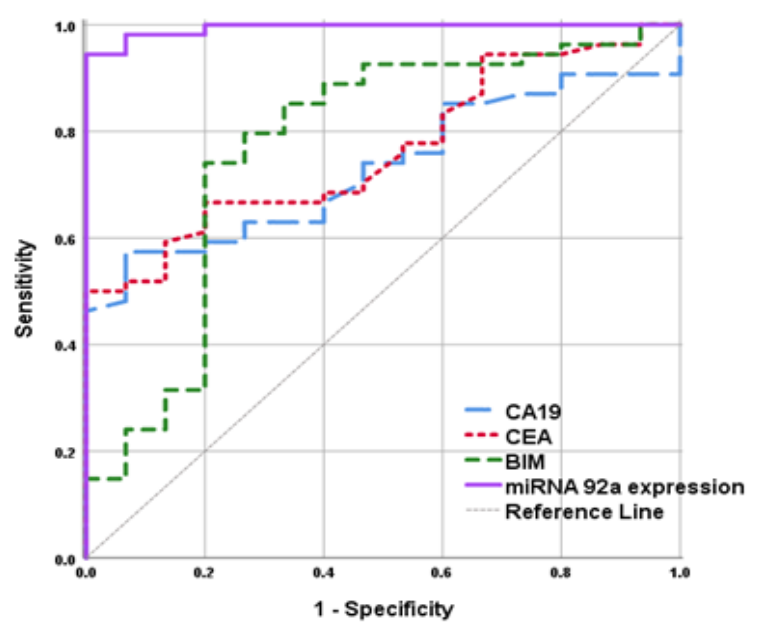

(B)

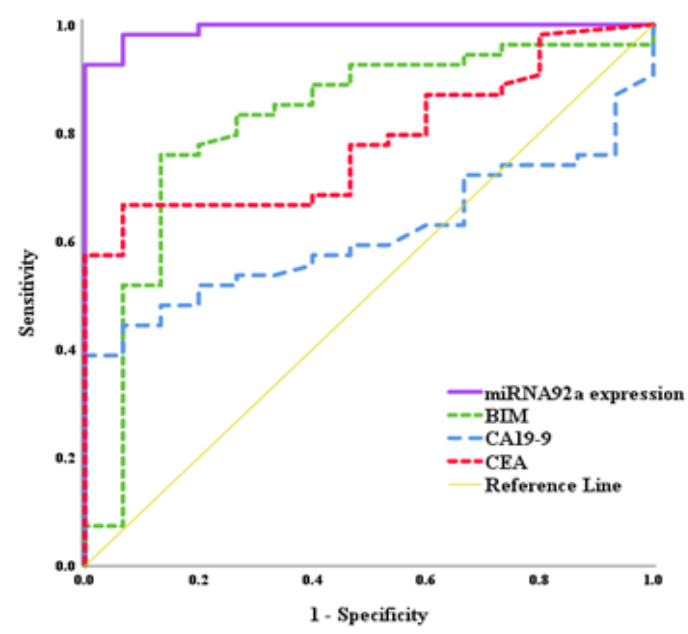

(C)

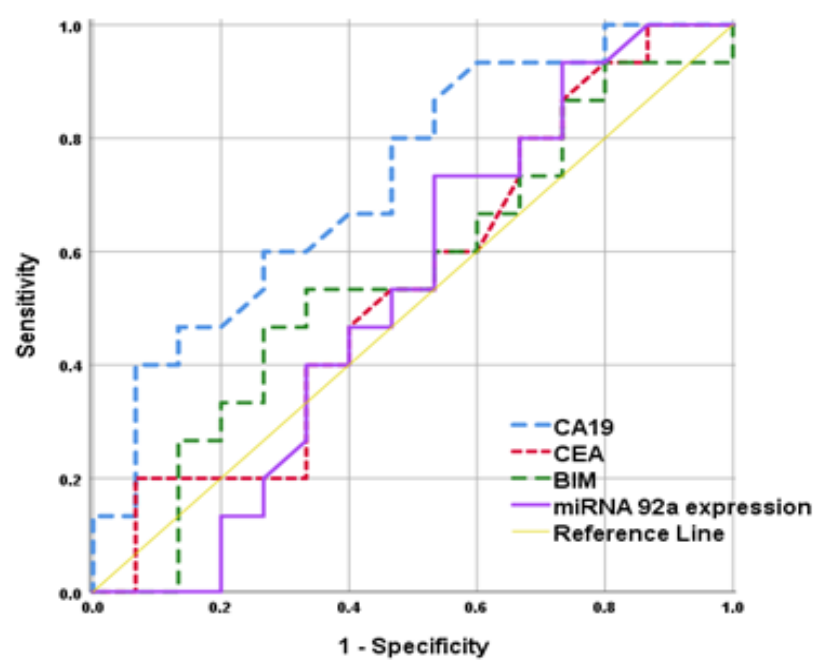

Figure 3. ROC Curves of Serum CA19-9, CEA, Bim Concentrations and Plasma miRNA92a Expression for Discrimination between Different Studied Groups. ROC curves of studied markers for discrimination between CRC patients and controls (A), CRC and adenoma patients (B), adenoma patients and controls (C).

significant downregulation in CRC patients $(75.5$; $17.9-2,062 \mathrm{ng} / \mathrm{ml})$ when compared with adenoma $(975.1$; $32.8-1,491 \mathrm{ng} / \mathrm{ml})$ and control $(602 ; 36.6-4768.8 \mathrm{ng} /$ $\mathrm{ml})$ groups $(\mathrm{p}=0.001, \mathrm{p}<0.001$ respectively) as shown in Figure 1 and Table 1. No significant difference was found in serum Bim levels between adenoma and control groups $(\mathrm{p}=0.604)$. Serum Bim showed significant lower levels in CRC advanced stages $(74.1 ; 17.9-2,062 \mathrm{ng} / \mathrm{ml})$ than in early stages $(169.2 ; 85.2-498 \mathrm{ng} / \mathrm{ml})(\mathrm{p}=0.03)$. Otherwise, No significant differences were found in serum Bim according to clinicopathologic features of CRC and adenoma patients as shown in Table 1 and Figure 2.

Receiver Operating Characteristic (ROC) Curve analysis

The diagnostic utility of plasma miR-92a expression was evaluated by Receiver Operating Characteristic (ROC) curve analysis. The results shown in Table 2 and Figure 3, suggested that plasma miR-92a is the highest discriminator of CRC from adenoma patients (cutoff=1.78; $\mathrm{AUC}=0.993 ; \mathrm{Sn}=92.6 \% ; \mathrm{Sp}=93.3 \%$ ) and control subjects (cutoff $=1.43 ; \mathrm{AUC}=0.994 ; \mathrm{Sn}=98.1 \%$; $\mathrm{Sp}=93.9 \%$ ).

Serum Bim showed the second highest diagnostic accuracy after miR-92a as it could differentiate CRC from colonic adenoma patients (cutoff $<126.1 \mathrm{ng} / \mathrm{ml}$; $\mathrm{AUC}=0.819 ; \mathrm{Sn}=75.9 \% ; \mathrm{sP}=86.7 \%$ ), and $\mathrm{CRC}$ from controls, (cutoff $<110.1 \mathrm{ng} / \mathrm{ml} ; \mathrm{AUC}=0.770 ; \mathrm{Sn}=85.2 \%$; $\mathrm{Sp}=66.7 \%)$.

While, serum Bim concentrations and plasma miRNA-92a expression showed failed AUCs (0.556, 0.529 respectively) and could not discriminate between adenoma and control groups.

Correlations of plasma miR-92a and serum Bim with clinicopathological features of CRC Group

Asian Pacific Journal of Cancer Prevention, Vol 23 
Table 2. Diagnostic Performance Features of Studied Markers in All Studied Groups

\begin{tabular}{|c|c|c|c|c|c|c|c|c|c|}
\hline Marker & Cut off & AUC & $\mathrm{Sn} \%$ & $\mathrm{Sp} \%$ & PPV\% & NPV\% & $95 \% \mathrm{CI}$ & Accuracy $(\%)$ & P value \\
\hline \multicolumn{10}{|c|}{ 1. Discrimination of CRC from the control group: } \\
\hline \multirow[t]{3}{*}{ CA19-9 (U/ml) } & 8.75 & 0.731 & 57.4 & 93.3 & 96.9 & 37.8 & $0.61-0.85$ & 65.2 & $P^{l}$ for $\mathrm{CEA}=0.679$ \\
\hline & & & & & & & & & $P^{l}$ for $\mathrm{Bim}=0.795$ \\
\hline & & & & & & & & & $P^{l}$ for miR-92a $<0.001 * *$ \\
\hline CEA (ng/ml) & 4.9 & 0.765 & 66.7 & 80 & 92.3 & 40 & $0.65-0.88$ & 69.6 & $P^{2}$ for $\mathrm{Bim}=0.919$ \\
\hline $\operatorname{Bim}(\mathrm{ng} / \mathrm{ml})$ & $<110.1$ & 0.77 & 85.2 & 66.7 & 90.2 & 55.6 & $0.62-0.92$ & 75.4 & $P^{2}$ for $\operatorname{miR}-92 \mathrm{a}=0.001 * *$ \\
\hline miR-92a & 1.43 & 0.994 & 98.1 & 93.9 & 98.1 & 93.2 & $0.98-1$ & 97.1 & $P^{3}=0.002^{* *}$ \\
\hline \multicolumn{10}{|c|}{ 2. Discrimination of CRC from the adenoma group: } \\
\hline \multirow[t]{3}{*}{ CA19-9 (U/ml) } & 15.9 & 0.612 & 44.4 & 93.3 & 96 & 31.8 & $0.48-0.74$ & 55.1 & $P^{l}$ for $\mathrm{CEA}=0.043^{*}$ \\
\hline & & & & & & & & & $P^{l}$ for $\mathrm{Bim}=0.041^{*}$ \\
\hline & & & & & & & & & $P^{I}$ for $\mathrm{miR}-92 \mathrm{a}<0.001^{* *}$ \\
\hline CEA (ng/ml) & 4.85 & 0.785 & 66.7 & 93.3 & 97.3 & 43.7 & $0.68-0.89$ & 72.5 & $P^{2}$ for $\mathrm{Bim}=0.873$ \\
\hline $\operatorname{Bim}(\mathrm{ng} / \mathrm{ml})$ & $<126.1$ & 0.819 & 75.9 & 86.7 & 90.2 & 55.6 & $0.69-0.95$ & 78.3 & $P^{2}$ for $\operatorname{miR}-92 \mathrm{a}=0.001 * *$ \\
\hline miR-92a & 1.78 & 0.993 & 92.6 & 93.3 & 98 & 77.8 & $0.979-1$ & 92.8 & $P^{3}=0.002^{* *}$ \\
\hline \multicolumn{10}{|c|}{ 3. Discrimination of adenoma from the control group: } \\
\hline \multirow[t]{3}{*}{ CA19-9 (U/ml) } & 6.8 & 0.727 & 60 & 73.3 & 69.2 & 64.7 & $0.55-0.91$ & 66.7 & $P^{l}$ for $\mathrm{CEA}=0.236$ \\
\hline & & & & & & & & & $P^{l}$ for $\operatorname{Bim}=0.176$ \\
\hline & & & & & & & & & $P^{l}$ for miR-92a $=0.196$ \\
\hline CEA (ng/ml) & $<4.1$ & 0.542 & 80 & 33.3 & 54.5 & 62.5 & $0.33-0.75$ & 56.7 & $P^{2}$ for $\operatorname{Bim}=0.865$ \\
\hline $\operatorname{Bim}(\mathrm{ng} / \mathrm{ml})$ & 943.1 & 0.556 & 53.3 & 66.7 & 61.5 & 58.8 & $0.34-0.77$ & 60 & $P^{2}$ for $\operatorname{miR}-92 \mathrm{a}=0.920$ \\
\hline miR-92a & 0.215 & 0.529 & 73.3 & 46.7 & 57.9 & 63.6 & $0.31-0.74$ & 60 & $P^{3}=0.944$ \\
\hline
\end{tabular}

AUC, area under ROC; ROC, receiver operating curve; $\mathrm{CI}$, confidence interval; PPV, positive predictive value; NPV, negative predictive value; $P^{I}$, comparison of AUCs versus CA19-9 AUC; $P^{2}$, comparison of AUCs versus CEA AUC; $P^{3}$, comparison of AUCs versus Bim AUC.

Statistical analysis using Spearman's correlation coefficient showed significant negative correlation between plasma miR-92a expression and serum Bim level both in adenoma and CRC groups ( $\mathrm{P}<0.001$ for both). Moreover, there was a significant positive correlation between plasma miR-92a expression and tumor staging $(\mathrm{P}=0.028)$. While, serum level of Bim showed a significant negative correlation with tumor staging $(\mathrm{P}=0.044)$.

Table 3. Correlations of miR-92a and Bim with Other Studied Parameters in CRC Patients

\begin{tabular}{lcccc}
\hline Parameter & \multicolumn{2}{c}{ Serum Bim } & \multicolumn{2}{c}{ Plasma miR-92a } \\
& $R$ & P value & $r$ & p value \\
\hline In CRC group: & & & & \\
Age & -0.004 & 0.98 & -0.127 & 0.359 \\
Tumor Size & 0.49 & 0.066 & -0.355 & 0.163 \\
Modified Dukes & -0.044 & 0.755 & 0.053 & 0.702 \\
Histological Grade & 0.079 & 0.572 & 0.121 & 0.383 \\
TNM staging & -0.299 & 0.028 & 0.275 & 0.044 \\
CA19-9 & -0.019 & 0.889 & -0.005 & 0.97 \\
CEA & -0.008 & 0.954 & 0.129 & 0.351 \\
Serum Bim & NA & NA & -0.95 & $<0.001$ \\
In Adenoma group: & & & & \\
CA19-9 & 0.025 & 0.93 & 0.339 & 0.216 \\
CEA & 0.07 & 0.804 & -0.222 & 0.426 \\
Serum Bim & - & - & -0.904 & $<0.001$ \\
\hline
\end{tabular}

$*$ P value $<0.05$ is significant
Otherwise, no significant correlations were found in serum Bim level and plasma miRNA 92a expression with age, tumor size, histological grading, and other serum markers in the studied groups as shown in Table 3.

\section{Discussion}

CRC is a heterogeneous carcinoma with markedly variable incidence and mortality rates globally (Ward et al., 2019). So, there is high need to find novel non-invasive biomarkers that can assist in identification of CRC in early stages, and help determine disease prognosis.

Our data showed that plasma miR-92a expression was significantly up-regulated in CRC patients when compared to both adenoma and control groups. This was in agreement with studies of Huang (2010); Liu (2013); Elshafei (2017); and Chen (2018), supporting that miR$92 \mathrm{a}$ is considered as an oncogene in CRC, and has the ability to promote tumor proliferation, migration and invasion.

This study results were also consistent with several studies performed on CRC tissues and stool samples. Wu (2012); and Lv (2016), reported that miR-92a expression was increased in tumor tissues, cell lines, and stools of CRC patients compared with that in corresponding adjacent normal tissues and stools of normal subjects.

Similarly, upregulation of miR-92a expression was reported in various malignancies including cervical cancer and osteosarcoma via targeting p21 and FBXW7 proteins, respectively (Su et al., 2017; Jiang et al., 2017), oral 
squamous cell carcinoma by targeting FOXP1 expression (Guo et al., 2018), and NSCLC via targeting PTEN (Lu et al., 2017).

miR-92a regulates cell survival mechanisms by acting on different target genes. As an oncogene, it promotes tumor proliferation, metastases, and angiogenesis via inhibiting tumor apoptosis by suppressing the action of multiple proteins such as Bim (Penzkofer et al., 2014). Thus, its upregulation is strongly implicated in carcinogenesis and progression.

To extend miR-92a study in CRC pathogenesis, Bim was studied as one of its molecular targets. Up to our knowledge, no research studies evaluated the diagnostic performance of circulating Bim in serum or plasma of CRC patients. We found that serum Bim levels in CRC patients were significantly lower in comparison to adenoma and control groups. This was in agreement with studies done on CRC tissue samples by Greenhough et al., (2010); Wang et al., (2008); and Tsuchida et al., (2011).

Regarding association of miR-92a with clinicopathologic parameters of CRC patients, our results revealed significant association between its high plasma expressions with advanced tumor stage. This can support its role in invasion, and progression of CRC by repressing Bim protein (Penzkofer et al., 2014).

There was also significant association between serum Bim low levels and advanced tumor stage. On the other side, no significant correlation was found between either plasma miR-92a or serum Bim and other clinicopathologic features of CRC patients including age, tumor size, histological grading, or other serum biomarkers.

In agreement with our findings, Tsuchida et al., (2011) revealed that the higher miR-92a staining score using in-situ hybridization, and decreased Bim expression were directly correlated with increased incidence of lymph node metastases (P value $<0.001$ for both). Similarly, Elshafei et al., (2017) concluded that serum miR-92a expression was positively correlated $(\mathrm{r}=0.671, \mathrm{p}=0.0001)$ only with advanced CRC stages. Other consistent studies showed significant positive correlation between miR-92a expression and advanced clinical and TNM staging with $(p=0.008)$ (Thiery et al., 2009); $(p=0.025)$ (Zhou et al., 2013); $(p=0.008)$ (Zhang et al., 2014); $(p=0.01)$ (Ke et al., 2015); $(\mathrm{p}=0.002)$ (Song et al., 2016); and $(\mathrm{p}=0.001)$ (Li et al., 2016).

In several opponent studies to our results, Huang et al., (2010); Wang and Jin (2011); Schee (2012); Faltejskova (2013); and Yang (2014), found no significant correlation between miR-92a expression and TNM staging ( $\mathrm{P}>0.05)$. Moreover, Sinicrope et al., (2008) concluded that there was no significant association of Bim expressions in CRC tissues with any clinicopathologic features.

Our study revealed significant negative correlation between plasma miR-92a expression and serum Bim in CRC and adenoma groups $(\mathrm{P}<0.001$ for both) and correlation coefficient $(r)=(-0.950,-0.904$ respectively $)$.

Several studies have implicated Bim as a target of miR92a in CRC development (Tsuchida et al., 2011; Elshafei et al., 2017), glioma tumorigenesis (Niu et al., 2012), and lymphomagenesis (Ventura et al., 2008; Xiao et al., 2008). The up-regulation of miR-92a expression directly represses Bim protein which relives its tumor-suppressing action and thought to have important role in promoted angiogenesis in CRC (Elshafei et al., 2017).

Our study results suggested that plasma miR-92a expression could discriminate $\mathrm{CRC}$ patients from colonic adenoma patients and controls with excellent AUC, sensitivity, and specificity.

Serum Bim showed promising discriminator between CRC and adenoma patients, with good AUC, sensitivity, and specificity. While, its discriminating power is fair between CRC and controls.

Several agreeing studies including comprehensive meta-analyses showed that miR-92a yielded diagnostic sensitivity ranging $(65.5 \%-89 \%)$, and diagnostic specificity ranging (64\%-89\%), and AUC ranging (0.7720.940 ) indicating its moderate to high diagnostic power as convenient non-invasive detection method of CRC (Liu et al., 2013; Yang et al., 2014; Jiang et al., 2019; Peng et al., 2019).

The inconsistent miR-92a expression pattern in $\mathrm{CRC}$ and controversies in its correlation with other clinicopathological features could be attributed to sample types variations in different studies (Clancy et al., 2015), various detection methods of miR-92a (Fendler et al., 2016), and difference in subjects ethnicity (Elshafei et al., 2017)

In conclusion, plasma miR-92a expression was significantly upregulated in CRC patients when compared to adenoma patients and controls, which strongly suggests its promising role as potential non-invasive and reliable biomarker assisting diagnosis of CRC. Also, its plasma expression was negatively correlated with serum Bim, which was downregulated in CRC patients, and this may explain the miR-92a molecular effect on increased risk of CRC. miR-92a overexpression and low serum Bim, were associated with late tumor stages. So, they could help as prognostic markers in CRC.

\section{Author Contribution Statement}

The professors: Samia Y. Akel, Amal Fawzy and Reham A. Elshimy planed and designed the research; Ahmed Kamal Zaki participated in conception, interpretation of laboratory data and practical work. Hebatallah Gamal El Din and Ahmed Kamal Zaki collected clinical data and performed statistical analysis. All authors - contributing to the study design, participated in writing and editing final version of the manuscript, read and approved the final manuscript.

\section{Acknowledgements}

The authors would like to thank chemists and technicians of the Clinical Chemistry Unit at NCI, Cairo University.

\section{Funding Statement}

This work was funded by NCI, Cairo University and was a part of Ahmed Kamal Zaki approved MD thesis.

\section{Ethical Approval and Consent}

Asian Pacific Journal of Cancer Prevention, Vol 23 
The study was approved by the IRB of NCI, Cairo University. A written consent was obtained from all subjects before their enrollment.

\section{Conflict of Interest}

The authors declare no conflict of interest.

\section{References}

Bader El Din NG, Ibrahim MK, El-Shenawy R, et al (2020). MicroRNAs expression profiling in Egyptian colorectal cancer patients. IUBMB Life, 72, 275-84.

Cekaite L, Eide PW, Lind GE, Skotheim RI (2016). MicroRNAs as growth regulators, their function and biomarker status in colorectal cancer. Oncotarget, 7, 6476-505.

Clancy C, Joyce MR, Kerin MJ (2015). The use of circulating microRNAs as diagnostic biomarkers in colorectal cancer. Cancer Biomark, 15, 103-13.

Elshafei A, Shaker O, Abd El-Motaal O, Salman T (2017). The expression profiling of serum miR-92a, miR-375, and miR-760 in colorectal cancer: An Egyptian study. Tumor Biol, 39, 1-14.

Faltejskova P, Bocanek O, Sachlova M, et al (2013). Circulating miR-17-3p, miR-29a, miR-92a and miR-135b in serum: Evidence against their usage as biomarkers in colorectal cancer. Cancer Biomarkers, 12, 199-204.

Fang LL, Wang XH, Sun BF, et al (2017). Expression, regulation and mechanism of action of the miR-17-92 cluster in tumor cells (Review). Int J Mol Med, 40, 1624-30.

Fendler A, Stephan C, Yousef GM, Kristiansen G, Jung K, (2016). The translational potential of microRNAs as biofluid markers of urological tumors. Nat Rev Urol, 13, 734-52.

Ferrari A, Neefs I, Hoeck S, Peeters M, Van Hal G (2021). Towards novel non-invasive colorectal cancer screening methods: A Comprehensive Review. Cancers, 13, 1820.

Greenhough A, Wallam CA, Hicks DJ, et al (2010). The proapoptotic $\mathrm{BH} 3$-only protein Bim is downregulated in a subset of colorectal cancers and is repressed by antiapoptotic COX-2/PGE(2) signalling in colorectal adenoma cells. Oncogene, 29, 3398-3410.

Guo J, Wen N, Yang S, Guan X, Cang S (2018). miR-92a regulates oral squamous cell carcinoma (OSCC) cell growth by targeting FOXP1 expression. Biomed Pharmacother, 104, P77-86.

Huang Z, Huang D, Ni S, et al (2010). Plasma microRNAs are promising novel biomarkers for early detection of colorectal cancer. Int J Cancer, 126, 118-26.

Jiang M, Li X, Quan X, Li X, Zhou B (2019). MiR-92a Family: A Novel Diagnostic Biomarker and Potential Therapeutic Target in Human Cancers. Front Mol Biosci, 6, 98.

Jiang X, Li X, Wu F, et al (2017). Overexpression of miR-92a promotes the tumor growth of osteosarcoma by suppressing F-box and WD repeat-containing protein7. Gene, 606, 10-6,

Li ML, Guan XF, Sun YQ, et al (2014). miR-92a family and their target genes in tumorigenesis and metastasis. Exp Cell Res, 323, 1-6.

Liu GH, Zhou ZG, Chen R, et al (2013). Serum miR-21 and miR-92a as biomarkers in the diagnosis and prognosis of colorectal cancer. Tumor Biol, 34, 2175-81.

Livak KJ, Schmittgen TD (2001). Analysis of relative gene expression data using real-time quantitative PCR and the $2^{-\triangle \Delta C T}$ method. Methods, 25, 402-8.

Lu C, Shan Z, Hong J, Yang L, (2017). MicroRNA-92a promotes epithelial-mesenchymal transition through activation of PTEN/PI3K/AKT signaling pathway in non-small cell lung cancer metastasis. Intl J Onc.;51: 235-44.
Lv H, Zhang Z, Wang Y, et al (2016). MicroRNA-92a Promotes Colorectal Cancer Cell Growth and Migration by Inhibiting KLF4. Oncol Res, 23, p283-90.

Mogilyansky E, Rigoutsos I (2013). The miR-17/92 cluster: a comprehensive update on its genomics, genetics, functions and increasingly important and numerous roles in health and disease. Cell Death Differ, 20, 1603-14.

Niu H, Wang K, Zhang A, et al (2012). miR-92a is a critical regulator of the apoptosis pathway in glioblastoma with inverse expression of BCL2L11. Oncol Rep, 28, 1771-7.

Peng Q, Shen Y, Lin K, et al (2019). Identification of microRNA$92 \mathrm{a}$ and the related combination biomarkers as promising substrates in predicting risk, recurrence and poor survival of colorectal cancer. J Cancer, 10, 3154-71.

Penzkofer D, Bonauer A, Fischer A, et al (2014). Phenotypic characterization of miR-92a-/- mice reveals an important function of miR-92a in skeletal development. PLoS One, 9, e101153.

Qadir MF, Klein D, Alvarez-Cubela S, et al (2019). The role of MicroRNAs in diabetes-related oxidative stress. Int $\mathrm{J} \mathrm{Mol}$ Sci, 20, 5423.

Schee K, Boye K, Abrahamsen TW, et al (2012). Clinical relevance of microRNA miR-21, miR-31, miR-92a, miR101, miR-106a and miR-145 in colorectal cancer. BMC Cancer, 12, 505.

Sinicrope FA, Rego RL, Okumura K, et al (2008). Prognostic Impact of Bim, Puma, and Noxa Expression in Human Colon Carcinomas. Clin Cancer Res, 14, 5810-8.

Su Z, Yang H, Zhao M (2017). MicroRNA-92a promotes cell proliferation in cervical cancer via inhibiting p21 expression and promoting cell cycle progression. Oncol Res, 25, 137-45.

Tsuchida A, Ohno S, Wu W, et al (2011). miR-92 is a key oncogenic component of the miR-17-92 cluster in colon cancer. Cancer Sci, 102, 2264-71.

Ventura A, Young AG, Winslow MM, et al (2008). Targeted deletion reveals essential and overlapping functions of the miR-17 through 92 family of miRNA clusters. Cell, 132, $875-86$.

Wang LC, Geng JS (2008). Expression of Bim gene in colorectal cancer. World Chin J Dig, 16, 4062-5.

Wang LG, Jin G (2011). Serum microRNA-29a is a promising novel marker for early detection of colorectal liver metastasis. Cancer Epidemiol, 36, 61-7.

Ward EM, Sherman RL, Henley SJ, et al (2019). Annual Report to the Nation on the Status of Cancer, Featuring Cancer in Men and Women Age 20-49 Years. J Natl Cancer Inst, 111, 1279

Wu CW, Ng SM, Dong YJ, et al (2012). Detection of miR-92a and miR-21 in stool samples as potential screening biomarkers for colorectal cancer and polyps. Gut, 61, 739-45.

Xiao C, Srinivasan L, Calado DP, et al (2008). Lymphoproliferative disease and autoimmunity in mice with increased miR-17-92 expression in lymphocytes. Nat Immunol, 9, 405-14.

Xuan Y, Yang H, Zhao L, et al (2015). MicroRNAs in colorectal cancer: small molecules with big functions. Cancer Lett, 360, 89-105.

Yang Y, Peng W, Tang T, et al (2014). MicroRNAs as promising biomarkers for tumor-staging: Evaluation of MiR21 MiR155 MiR29a and MiR92a in Predicting Tumor Stage of Rectal Cancer. Asian Pac J Cancer Prev, 15, 5175-80.

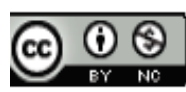

This work is licensed under a Creative Commons AttributionNon Commercial 4.0 International License. 